\title{
Inhibition of Glycogen Synthase Kinase 3 Accelerated Liver Regeneration after Acetaminophen-Induced Hepatotoxicity in Mice
}

\author{
Bharat Bhushan, Samikshya Poudel, Michael W. Manley, Jr, Nairita Roy, and Udayan Apte
}

From the Department of Pharmacology, Toxicology, and Therapeutics, University of Kansas Medical Center, Kansas City, Kansas

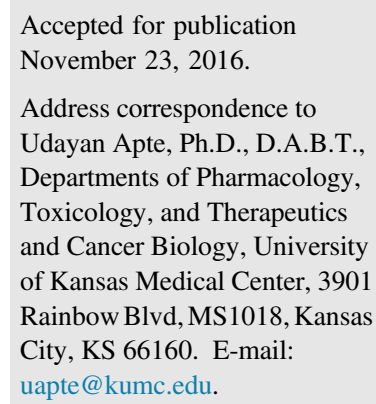

\begin{abstract}
Overdose of acetaminophen (APAP) is the leading cause of acute liver failure (ALF) in the United States. Timely initiation of compensatory liver regeneration after APAP hepatotoxicity is critical for final recovery, but the mechanisms of liver regeneration after APAP-induced ALF have not been extensively explored yet. Previous studies from our laboratory have demonstrated that activation of $\beta$-catenin signaling after APAP overdose is associated with timely liver regeneration. Herein, we investigated the role of glycogen synthase kinase 3 (GSK3) in liver regeneration after APAP hepatotoxicity using a pharmacological inhibition strategy in mice. Treatment with specific GSK3 inhibitor (L803-mts), starting from 4 hours after $600 \mathrm{mg} / \mathrm{kg}$ dose of APAP, resulted in early initiation of liver regeneration in a dose-dependent manner, without modifying the peak regenerative response. Acceleration of liver regeneration was not secondary to alteration of APAP-induced hepatotoxicity, which remained unchanged after GSK3 inhibition. Early cell cycle initiation in hepatocytes after GSK3 inhibition was because of rapid induction of cyclin D1 and phosphorylation of retinoblastoma protein. This was associated with increased activation of $\beta$-catenin signaling after GSK3 inhibition. Taken together, our study has revealed a novel role of GSK3 in liver regeneration after APAP overdose and identified GSK3 as a potential therapeutic target to improve liver regeneration after APAP-induced ALF. (Am J Pathol 2017, 187: 543-552; http://dx.doi.org/10.1016/ j.ajpath.2016.11.014)
\end{abstract}

Liver injury caused by acetaminophen (APAP) overdose is the leading cause of acute liver failure (ALF) in the western world, accounting for nearly half of all of the ALF cases. ${ }^{1,2}$ Despite decades of research, current treatment options after APAP overdose are extremely limited. $\mathrm{N}$-acetylcysteine is the current standard of care for APAP overdose patients, which is effective only at an early stage. ${ }^{3,4}$ Mechanisms of liver injury after APAP overdose have been extensively investigated in the past to develop novel therapeutic strategies. However, many patients seek medical attention late after liver injury is already established and difficult to manipulate. ${ }^{5}$ Liver injury after APAP overdose is subsequently followed by compensatory liver regeneration, which is critical for recovery. ${ }^{6-8}$ Previous studies have demonstrated that liver regeneration is a critical determinant of survival after APAP overdose. ${ }^{9-12}$ Liver regeneration is a parameter that can be potentially modulated even at a late stage in pathogenesis of APAP-induced ALF, and stimulating liver regeneration in patients of APAP-induced ALF holds a great therapeutic potential. However, development of novel regenerative therapies for ALF is hampered because the mechanisms of liver regeneration after APAP-induced ALF have not been extensively explored yet. Mechanisms of liver regeneration are mostly studied in the partial hepatectomy (PH) model, ${ }^{13}$ which is considerably different from APAP hepatotoxicity, the model of clinical ALF. ${ }^{6}$

Supported by NIH grants 8P20 GM103549, 5T32ES007079-34, and R01DK098414, and American Association for the Study of Liver Diseases/ Acute Liver Failure Liver Scholar Award (U.A.).

Disclosures: None declared. 
Glycogen synthase kinase 3 (GSK3) is a ubiquitously expressed serine/threonine protein kinase, which is constitutively active in cells under resting state and primarily regulated by inactivation. ${ }^{14}$ In mammals, GSK3 exists in two isoforms, GSK3 $\alpha$ and GSK3 $\beta$, which share $98 \%$ sequence homology in the kinase domain and have many redundant functionalities. ${ }^{14}$ GSK3 is downstream of several major signaling pathways, including growth factors, insulin, and canonical Wnt signaling, which negatively regulate GSK3 activity. GSK3 activity is primarily regulated by phosphorylation-mediated inactivation, but other methods of inactivation, such as sequestration and subcellular localization, are also known. ${ }^{15}$ Initially discovered to regulate glycogen synthase, GSK3 is now known to regulate a wide spectrum of proteins and affect several cellular functionalities, including proliferation, differentiation, and survival. ${ }^{15}$ GSK3 functions by phosphorylation-mediated inactivation/ degradation of its substrates. As a subunit of the $\beta$-catenin destruction complex, GSK3 causes phosphorylationmediated targeting of $\beta$-catenin for proteosomal degradation, thus negatively regulating $\beta$-catenin signaling. ${ }^{16}$ Apart from $\beta$-catenin, GSK3 can regulate many other cell proliferation mediators, such as cyclin D1, c-Myc, c-Jun, and eIF2B. ${ }^{17}$

Inhibitors of GSK3 can induce in vitro expansion of mouse primary hepatocytes ${ }^{18}$ and facilitate survival signaling via activation of $\beta$-catenin in them. ${ }^{19}$ Furthermore, GSK3 negatively regulates $\beta$-catenin, which plays a critical role in liver regeneration. ${ }^{20-23}$ Interestingly, other studies indicate that GSK3 may play a proregenerative role after PH. ${ }^{24-26}$ Treatment with GSK3 inhibitor or siRNAmediated down-regulation of GSK3 impaired liver regeneration in mice and rats after $\mathrm{PH}^{24-26}$ Furthermore, antisense oligonucleotide-mediated silencing of GSK3 has reported to inhibit APAP-induced injury, attributed to the role of GSK3 in initiation of APAP toxicity. ${ }^{27}$ However, the role of GSK3 in compensatory liver regeneration after APAP-induced liver injury is not known. We developed a novel incremental dose model in mice to study mechanisms of liver regeneration after APAP-induced ALF, ${ }^{6}$ which revealed several signaling pathways involved in liver regeneration after APAP overdose. These data also showed that activation of $\beta$-catenin signaling was associated with stimulation of liver regeneration after APAP overdose. $^{6}$ Furthermore, overexpression of $\beta$-catenin resulted in improved liver regeneration after APAPinduced liver injury. ${ }^{6}$ Herein, we investigated the direct role of GSK3 in compensatory liver regeneration after APAP overdose using a pharmacological inhibitor of GSK3. On the basis of our previous findings, we hypothesized that inhibition of GSK3 after APAP treatment will result in enhanced liver regeneration. ${ }^{6}$ Herein, we report that treatment with L803-mts, a selective GSK3 inhibitor, accelerates liver regeneration after APAP overdose via early activation of $\beta$-catenin and induction of cyclin D1.

\section{Materials and Methods}

\section{Animals, Treatments, and Tissue Harvesting}

Eight-week-old C57BL/6J mice were purchased from Jackson Laboratories (Bar Harbor, ME) and used in all of the studies. All animals were housed in Association for Assessment and Accreditation of Laboratory Animal Careaccredited facilities at the University of Kansas Medical Center under a standard 12-hour light/dark cycle with access to chow and water ad libitum. All studies were approved by The Institutional Animal Care and Use Committee at the University of Kansas Medical Center. APAP (Sigma, St. Louis, MO) was dissolved in warm $0.9 \%$ saline and administered i.p. Mice were fasted overnight before APAP administration. For study with incremental doses of APAP, mice $(n=3$ to 8$)$ were treated with either 300 or $600 \mathrm{mg} / \mathrm{kg}$ APAP, and sacrificed at $0,3,6,12,24,48,72$, and 96 hours after APAP treatment. For GSK3 inhibition studies, L803mts/N-myristol-GKEAPPAPPQS(p)P (Genemed Synthesis, San Francisco, CA) was used. L803-mts is a substratecompetitive fatty acid-conjugated peptide inhibitor of GSK3. It contains a phosphorylated site that specifically mimics primed GSK3 substrate, providing it selectivity. Selectivity is the major issue for protein kinase inhibitors, which are mostly ATP competitive. ${ }^{28}$ Furthermore, the fatty acid moiety of the inhibitor provides cell permeability and stability in vivo. ${ }^{28}$ GSK3 inhibitor (GSK3i) was dissolved in warm phosphate-buffered saline and administered 4 hours after APAP $(600 \mathrm{mg} / \mathrm{kg})$ treatment, followed by treatments every 24 hours until sample collection. For preliminary study, GSK3i was administered at doses of 400 and $800 \mathrm{nmol}$ per mouse, and animals ( $n=3$ to 4 ) were euthanized 24 hours after APAP treatment. In the following studies, $800 \mathrm{nmol} /$ mouse dose of GSK3i was administered, and mice $(n=4$ to 12) were sacrificed at 18, 24, 48, and 72 hours after treatment with APAP. Euthanasia was performed by cervical dislocation under isoflurane anesthesia, and blood and livers were collected. Serum samples were obtained from the blood and used for further analysis. Liver sections were prepared for histological analysis, as described previously. ${ }^{29}$

\section{Histological Analysis and Serum ALT Measurement}

Paraffin-embedded liver sections ( $4 \mu \mathrm{m}$ thick) were used for hematoxylin and eosin staining and scored for percentage necrotic area. Serum alanine aminotransferase (ALT) was measured using the Infinity ALT kit (ThermoFisher Scientific, Pittsburgh, PA), according to the manufacturer's protocol.

\section{Immunohistochemistry}

Paraffin-embedded liver sections $(4 \mu \mathrm{m}$ thick) were used for immunohistochemical detection. Proliferating cell nuclear antigen (PCNA) staining was performed for cell 
proliferation analysis, as described before. ${ }^{29}$ Anti-PCNA antibody was purchased from Cell Signaling Technologies (Danvers, MA). Biotinylated secondary antibody was purchased from Jackson Immunoresearch (West Grove, PA).

\section{Protein Isolation and Western Blot Analysis}

Total cell lysate was prepared from frozen liver tissues, as described previously. ${ }^{30}$ Protein estimation and Western blot analysis were performed using individual or pooled samples of protein extracts, as previously described in detail. ${ }^{29}$ All primary and secondary antibodies were obtained from Cell Signaling Technologies, unless stated otherwise. Active $\beta$-catenin antibody was purchased from EMD Millipore (Billerica, MA). ImageJ software version 1.50 (NIH, Bethesda, MD; http://imagej.nih.gov/ij) was used for densitometric analysis of Western blot images.

\section{Statistical Analysis}

Data presented in the form of bar/line graphs show means \pm SEM. Significant difference between two groups was determined using $t$-test and between three or more groups using one-way analysis of variance with Tukey's post hoc test. Difference between groups was considered statistically significant at $P<0.05$. Kaplan-Meier curves were used for survival analysis, and statistical significance was measured using the log-rank test.

\section{Results}

Inactivation of $\beta$-Catenin Was Associated with Impaired Liver Regeneration after Higher Dose of APAP

Our previous work established an incremental dose model to study mechanisms of liver regeneration after APAP-induced ALF. ${ }^{6}$ Two doses of APAP (300 and $600 \mathrm{mg} / \mathrm{kg}$ ) were administered in separate groups of mice. ${ }^{6}$ Both the doses caused extensive liver injury, but injury was followed by robust compensatory liver regeneration at the lower dose, leading to recovery. ${ }^{6}$ In contrast, at the higher dose, liver regeneration was lower and delayed, leading to decreased recovery. ${ }^{6}$ Interestingly, activation of $\beta$-catenin signaling was positively correlated with compensatory liver regeneration after APAP overdose. ${ }^{6}$ In the current study, phosphorylation of $\beta$-catenin was examined after incremental doses of APAP (300 and $600 \mathrm{mg} / \mathrm{kg}$ ) in mice over an extended time course up to 96 hours (Figure 1, A and B). Phosphorylation of $\beta$-catenin was examined at Thr41/Ser33/ Ser37 residues. These sites are known to be specifically phosphorylated by GSK3, ultimately leading to proteosomal-mediated degradation and inactivation of $\beta$-catenin. Thus, phosphorylation of these sites on $\beta$-catenin is a measure of $\beta$-catenin inactivation by GSK3 activity. Interestingly, a rapid increase in phospho $\beta$-catenin (Ser33/ Ser37/Thr41) was observed at the earliest investigated time point of 3 hours and peaked at approximately 6 to 12 hours
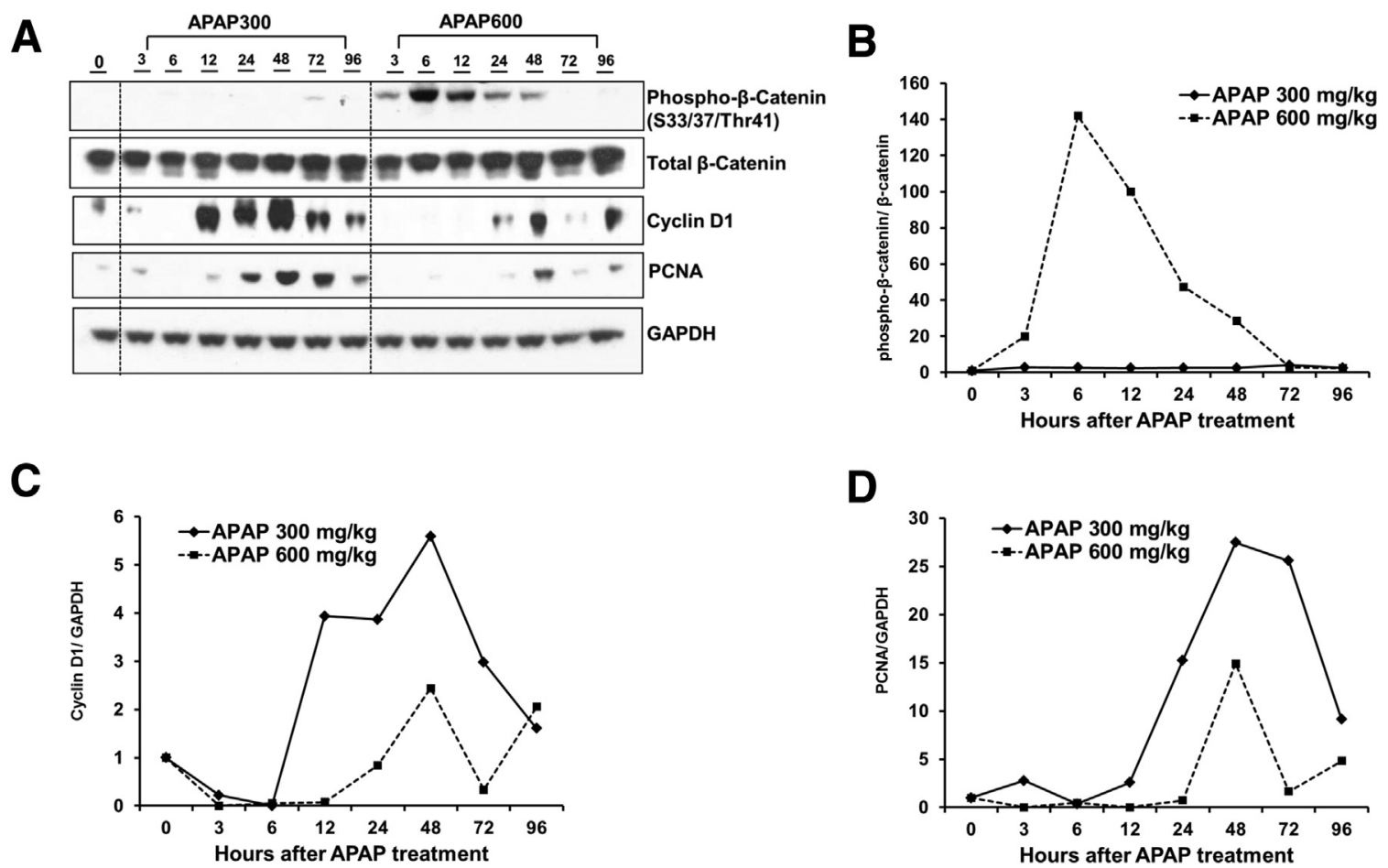

Figure 1 Rapid inactivation of $\beta$-catenin is associated with impaired liver regeneration at higher dose of APAP. A: Western blot analysis of phospho- $\beta$ catenin (Ser33/Ser37/Thr41), total $\beta$-catenin, cyclin D1, and proliferating cell nuclear antigen (PCNA) in total cell lysate of liver at various time points after administration of 300 and $600 \mathrm{mg} / \mathrm{kg}$ APAP in mice. B-D: Densitometric analysis showing $\beta$-catenin inactivation (phospho- $\beta$-catenin/ $\beta$-catenin; B), cyclin D1 (C), and PCNA protein expression (D) on the basis of Western blot images shown in A. GAPDH, glyceraldehyde-3-phosphate dehydrogenase. 
after treatment with a high dose of APAP. $\beta$-Catenin remained remarkably phosphorylated up to 48 hours, but phosphorylation gradually decreased with time and reached around basal levels at 72 and 96 hours. Phosphorylation of $\beta$-catenin did not appear to be remarkably altered at any of the investigated time points after a lower dose of APAP (Figure 1, A and B). As reported previously, ${ }^{6}$ increase in expression of PCNA, which is a maker of cell proliferation, was remarkably lower and delayed at a higher dose compared to a lower dose (Figure 1, A and D). A similar pattern was observed for protein expression of core cell cycle protein, cyclin D1, which governs entry into the cell cycle (Figure 1, A and C). These data indicate that inactivation of $\beta$-catenin by GSK3 correlates with delayed and impaired liver regeneration after severe APAP overdose.

\section{Pharmacological Inhibition of GSK3 Caused Dose-Dependent Improvement in Proliferation Parameters after a High Dose of APAP}

In the initial dose-finding study, L803-mts, a highly selective GSK $3 i^{28}$ was administered 4 hours after treatment with a high dose of APAP $(600 \mathrm{mg} / \mathrm{kg})$ in mice. All analysis was performed 24 hours after APAP treatment. Two doses of GSK3i (400 and $800 \mathrm{nmol}$ ) were used on the basis of previous in vivo studies in mice. ${ }^{28,31}$ APAP treatment caused significant liver necrosis in the centrilobular region and elevation of serum ALT levels (Figure 2, A and B). APAP-mediated liver injury was not altered by treatment with any of the two doses of GSK3i, as indicated by histopathological analysis of liver sections (Figure 2A) and serum ALT levels (Figure 2B). Next, cyclin D1 and PCNA protein expression levels were studied as a measure of liver regeneration using Western blot analysis. Despite similar injury, GSK3i treatment caused a significant increase in both cyclin D1 and PCNA protein expression at 24 hours after APAP treatment (Figure 2, C-E). Furthermore, an increase in expression of these proteins was dose dependently higher after treatment with $800 \mathrm{nmol}$ compared to $400 \mathrm{nmol}$ dose of GSK3i. The $800 \mathrm{nmol}$ dose of GSK3i was selected for the following comprehensive time course analysis of liver injury and regeneration.

\section{Inhibition of GSK3 Did Not Alter APAP-Induced Hepatotoxicity}

Next, 800 nmol of GSK3i was administered starting from 4 hours after treatment with $600 \mathrm{mg} / \mathrm{kg}$ APAP in mice. Liver injury was studied over a time course of 18 to 72 hours to cover both peak toxicity and liver regeneration time points. Liver injury was studied using histopathological analysis of hematoxylin and eosin-stained liver sections and serum ALT levels (Figure 3, A-C). APAP caused extensive liver necrosis (approximately 50\% necrotic area) at 18 hours after APAP treatment (Figure 3, A and B). Liver necrosis was sustained at all later time points up to 72 hours, as reported previously for a high dose of APAP treatment. ${ }^{6}$ Treatment with GSK3i did not significantly alter APAP-induced liver necrosis at any of the investigated time points (Figure 3,
A

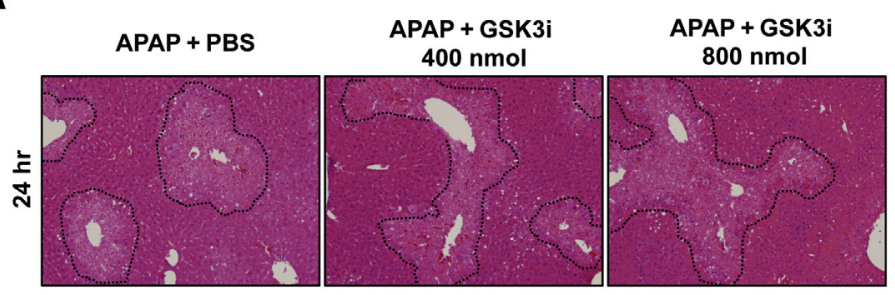

D

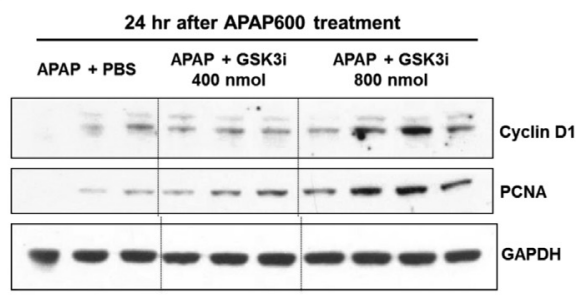

B

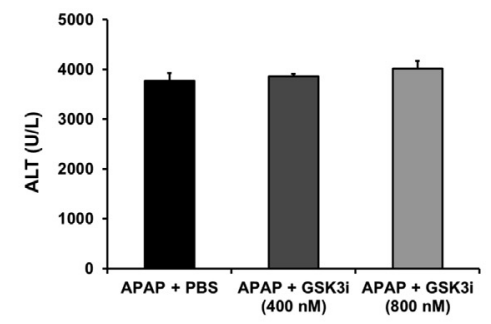

E

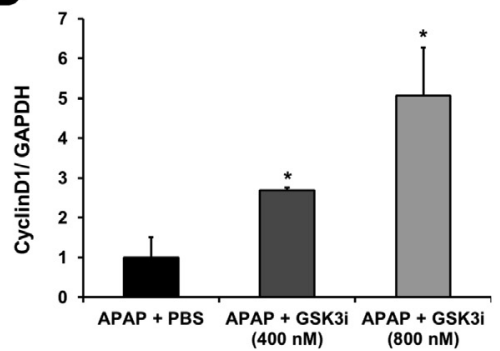

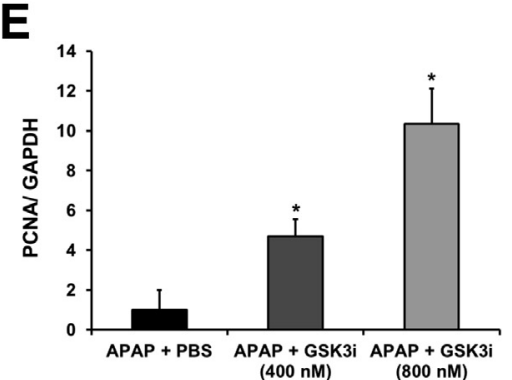

$(400 \mathrm{nM}) \quad(800 \mathrm{nM})$

Figure 2 Pharmacological inhibition of GSK3 caused dose-dependent improvement in proliferation parameters. A: Representative photomicrographs of hematoxylin and eosin-stained liver sections with necrotic areas outlined with dotted lines. B: Serum alanine aminotransferase (ALT) levels. C-E: Western blot analysis of cyclin D1 and proliferating cell nuclear antigen (PCNA) in liver lysate (C), with their densitometric analysis shown (D and E, respectively). All samples were collected at 24 hours after treatment with $600 \mathrm{mg} / \mathrm{kg}$ APAP. Vehicle [phosphate-buffered saline (PBS)] and L803-mts (400 and $800 \mathrm{nmol}$ per mouse) were administered 4 hours after treatment with APAP. ${ }^{*} P<0.05$ versus vehicle-treated group. GAPDH, glyceraldehyde-3-phosphate dehydrogenase; GSK3i, GSK3 inhibitor. 
A

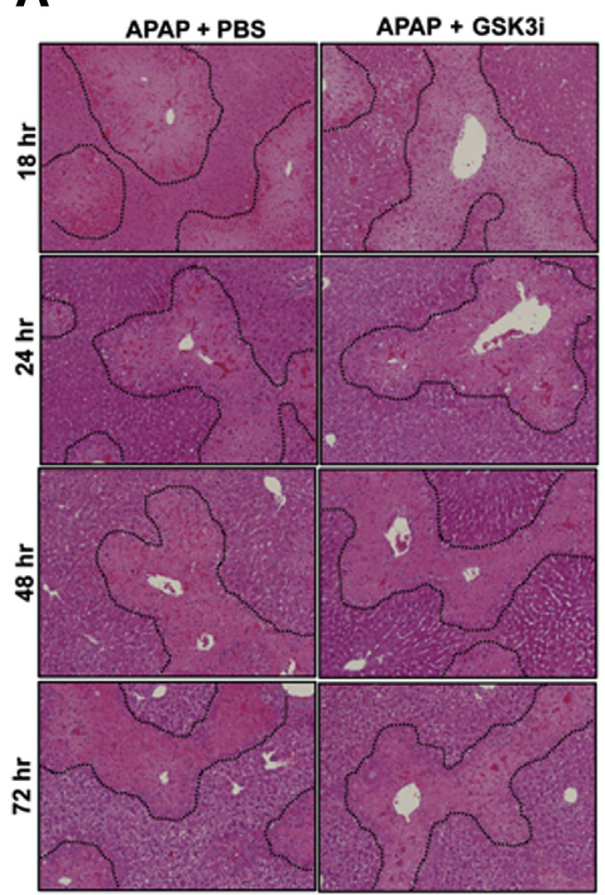

B

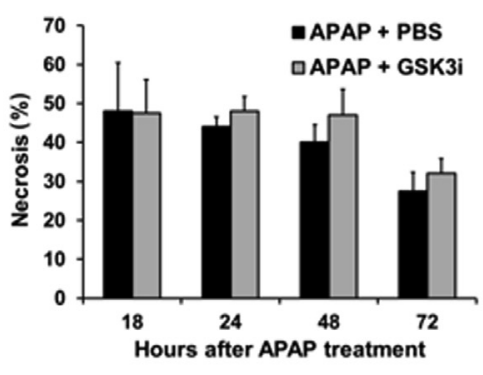

C

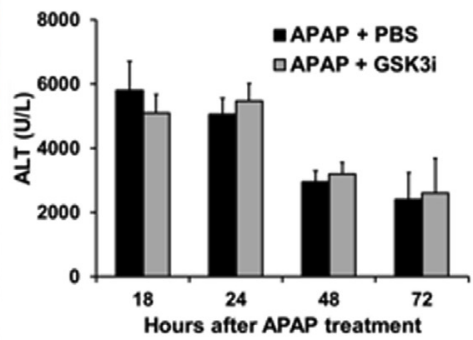

Figure 3 Inhibition of GSK3 did not alter APAPinduced hepatotoxicity. A: Representative photomicrographs of hematoxylin and eosin (H\&E)-stained liver sections with necrotic areas outlined in dotted lines. B: Percentage necrosis area on the basis of H\&E-stained liver sections. C: Serum alanine aminotransferase (ALT) levels. D: Survival analysis of mice treated with $600 \mathrm{mg} / \mathrm{kg}$ APAP, followed by treatment with L803-mts $(800 \mathrm{nmol}$ per mouse) or phosphate-buffered saline (PBS). L803-mts or PBS was administered 4 hours after APAP, followed by every 24 hours until sample collection. All samples were collected at 18, 24, 48, and 72 hours after APAP treatment, and survival was recorded up to 120 hours after APAP treatment. $n=4-6(\mathbf{A}-\mathbf{C}) ; n=10$ (D). GSK3i, GSK3 inhibitor.

D

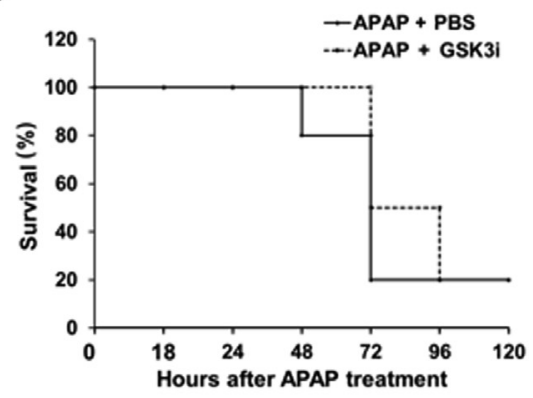

A and B). Furthermore, serum ALT analysis corroborated the histopathological results (Figure 3C). Some decrease in serum ALT levels with time was observed in both the groups, despite sustained necrotic areas. This is expected because of the limited half-life of ALT in serum and is consistent with our previous report. ${ }^{6}$ Next, we investigated the effect of GSK3i treatment on survival after APAP overdose (Figure 3D). No mortality was observed in any of the two groups up to 24 hours after APAP treatment; $20 \%$ mortality was observed in the APAP-alone treatment group at 48 hours, which remarkably increased to $80 \%, 72$ hours onwards. In contrast, no mortality was observed in the GSK3i-treated group at 48 hours, and 50\% mortality was observed at 72 hours, which increased to $80 \%$ beyond 96 hours (Figure 3D). Although our data indicated some delay in mortality after GSK3i treatment, no statistically significant difference was observed in overall survival between the two groups.

\section{Inhibition of GSK3 Caused Early Hepatocyte Proliferation and Liver Regeneration after APAP Overdose}

Next, we investigated the effect of GSK3 inhibition on compensatory liver regeneration after APAP-induced liver injury. Liver regeneration was studied by immunohistochemical analysis of PCNA over the time course of 18 to 72 hours after APAP administration, with or without treatment of GSK3i (Figure 4). Few PCNA-positive cells were observed at 18 and 24 hours after $600 \mathrm{mg} / \mathrm{kg}$ APAP treatment, consistent with our previous report. ${ }^{6}$ A significant number of positive cells were observed only at 48 and
72 hours in the APAP-alone treated group. Interestingly, GSK3 inhibition caused early entry into the cell cycle, with PCNA-positive cells observed surrounding the necrotic zones even at 18 hours. A remarkable increase in number of PCNA-positive cells was observed at 24 hours in the GSK3i-treated group. However, the number of PCNApositive cells was comparable to the APAP-alone treated group at 48 and 72 hours (Figure 4). In both the groups, PCNA-positive cells were observed predominantly in the areas adjacent to the necrotic zones (Figure 4 and Supplemental Figure S1). Western blot analysis corroborated PCNA immunohistochemistry data (Figure 5, A and C, and Supplemental Figure S2). PCNA expression increased starting from 24 hours in the GSK3i-treated group, whereas it increased only after 48 hours in the APAP-alone treated group. A significantly higher number of PCNA-positive cells and higher PCNA protein expression were observed at 24 hours after APAP administration in the GSK3i-treated group. These data demonstrated that inhibition of GSK3i resulted in an early liver regeneration response after APAP-induced liver injury.

\section{GSK3 Inhibition Caused Early and Sustained Expression of Cyclin D1 after APAP Overdose}

Next, we sought to investigate the mechanism of early increase in liver regeneration after GSK3 inhibition. Induction of cyclin D1 is a critical limiting factor that controls entry into the cell cycle and commitment to cell cycle progression in hepatocytes. ${ }^{32}$ Cyclin D1 protein expression was threefold higher in the GSK3i-treated group at 18 hours 


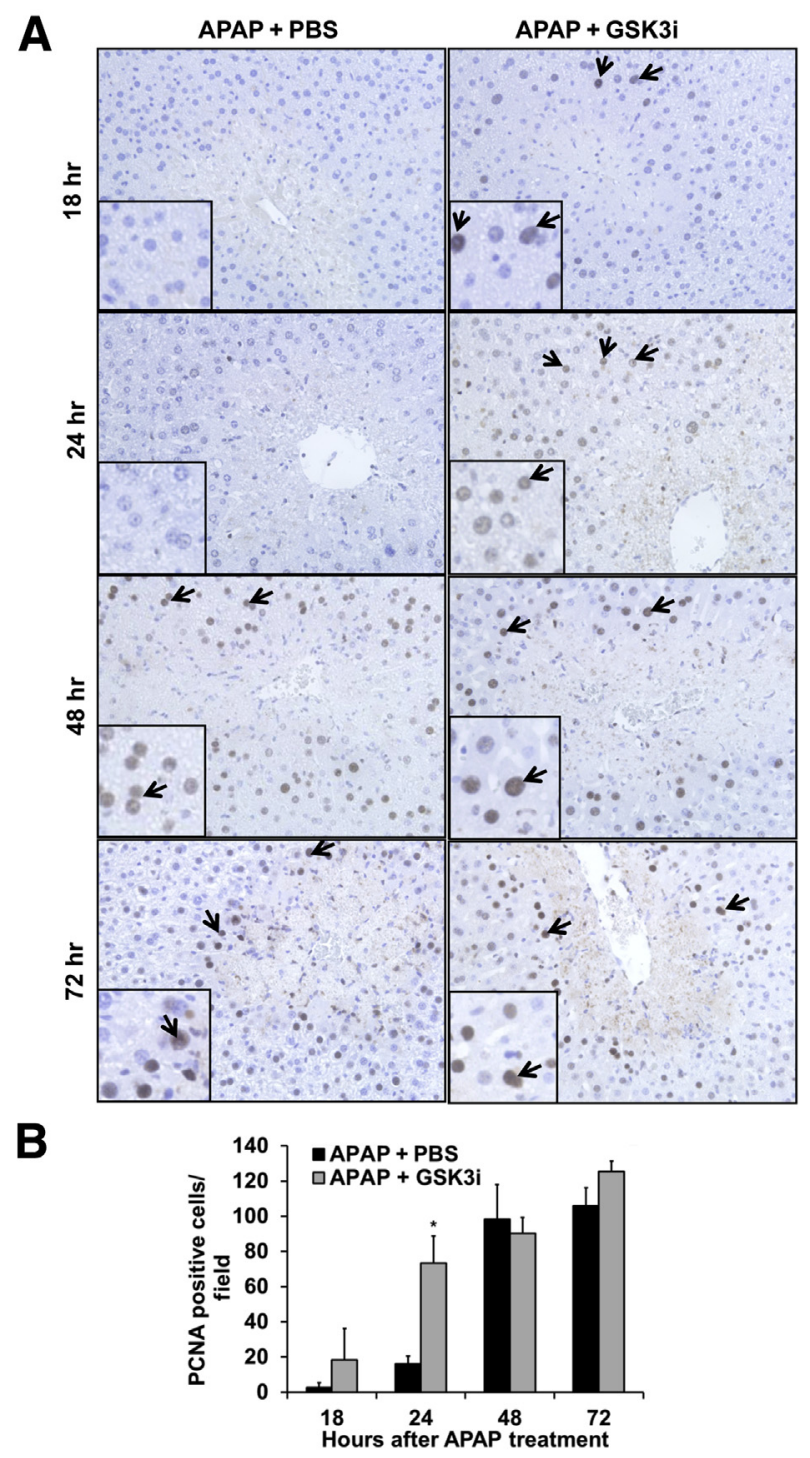

Figure 4 Inhibition of GSK3 caused early hepatocyte proliferation and liver regeneration after APAP overdose. A: Representative photomicrographs of proliferating cell nuclear antigen (PCNA)-stained liver sections, with arrows indicating cells with nuclear PCNA staining (brown). Insets show PCNA staining in higher magnification. B: Total number of PCNApositive cells per high-power field. Liver sections were obtained from mice treated with $600 \mathrm{mg} / \mathrm{kg}$ APAP, followed by treatment with L803-mts (800 nmol per mouse) or phosphate-buffered saline (PBS). L803-mts or PBS was administered 4 hours after APAP, followed by every 24 hours until sample collection. All samples were collected at 18, 24, 48, and 72 hours after APAP treatment. ${ }^{*} P<0.05$ versus APAP + PBS. Original magnification, $\times 200$ (A, main images); $\times 400$ (A, insets) GSK3i, GSK3 inhibitor.

after APAP and eightfold higher at 24 hours compared to the APAP-alone treated group (Figure 5, A and B). This correlated with early hepatocyte proliferation after GSK3 inhibition, which started at 24 hours. Furthermore, cyclin D1 expression increased with time in the GSK3i-treated group. Increase in cyclin D1 expression was also observed in the APAP-alone treated group but at delayed time points of 48 and 72 hours. However, cyclin D1 expression consistently remained remarkably higher in the GSK3i- treated group at all of the investigated time points compared to the APAP-alone treated group (Figure 5, A and B, and Supplemental Figure S2).

\section{Inhibition of GSK3 Resulted in Increased Activation of $\beta$-Catenin after APAP 0verdose}

Finally, we investigated the signaling mechanism(s) involved in early increase in cyclin D1 expression and increase in liver regeneration after GSK3 inhibition. GSK3 is known to negatively regulate $\beta$-catenin signaling, ${ }^{14}$ and we reported previously that stimulation of $\beta$-catenin signaling can improve liver regeneration after APAP-induced liver injury. ${ }^{6}$ Therefore, we investigated the effect on $\beta$-catenin signaling in our study. We analyzed the earliest time point of 18 hours that preceded a robust increase in liver regeneration response in the GSK3i-treated group. GSK3 causes phosphorylation of $\beta$-catenin at Thr41, Ser33, and Ser37, ultimately leading to proteosomal-mediated degradation of $\beta$-catenin. ${ }^{14}$ Phosphorylation of $\beta$-catenin was found to be significantly decreased in the GSK3i-treated group, 18 hours after APAP administration, as studied using antibody that specifically recognizes Thr41, Ser33, or Ser37 phosphorylation of $\beta$-catenin (Figure 6, A and B). This was corroborated with a fivefold increase in active $\beta$-catenin levels (dephosphorylated form) in the GSK3i-treated group (Figure 6, A and C). Total $\beta$-catenin levels were not altered by GSK3i treatment (Figure 6A). Stable (unphosphorylated) $\beta$-catenin translocates to the nucleus and induces its target genes, which includes cell proliferation genes, such as cyclin D1. Increase in $\beta$-catenin signaling was consistent with observed early increase in cyclin D1 levels at 18 hours after APAP treatment in the GSK3i group (Figure 5, A and B, and Figure 6, A and D). Cyclin D1 binds with cyclin-dependent kinase 4 and causes its activation, which then phosphorylates $\mathrm{Rb}$ protein, ultimately leading to induction of several key cell cycle genes. ${ }^{32}$ Furthermore, we observed increased phosphorylation of Rb protein in the GSK3i group at 18 hours after APAP treatment, corresponding with increased cyclin D1 expression (Figure 6, A and E). Thus, our results demonstrated that early activation of $\beta$-catenin after GSK3i inhibition might be involved in early entry into the cell cycle and accelerated liver regeneration after APAP-induced liver injury.

\section{Discussion}

Although mechanisms of APAP hepatotoxicity have been extensively studied, leading to considerable progress in our knowledge, mechanisms of compensatory liver regeneration that follows liver injury after APAP overdose have just begun to be explored, which have great potential to be used to find novel therapeutic targets. ${ }^{6}$ Herein, we investigated the role of GSK3 in liver regeneration after APAP-induced hepatotoxicity, which is currently unknown. 
A

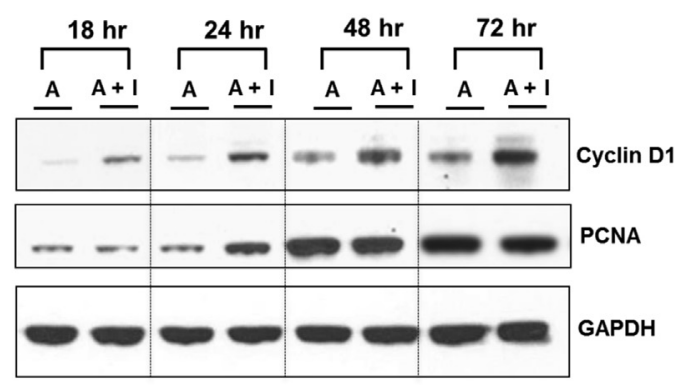

B
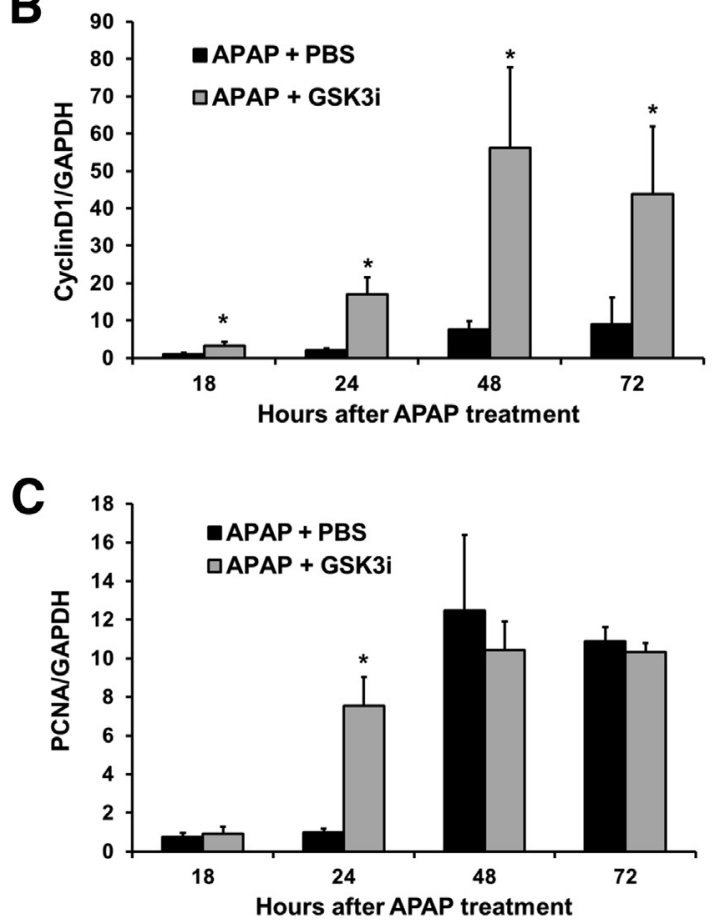

Figure 5 GSK3 inhibition caused early expression of cyclin D1 and proliferating cell nuclear antigen (PCNA) after APAP overdose. Western blot analysis of cyclin D1 and PCNA in pooled total cell lysate (A) with their densitometry on the basis of analysis of individual samples (B and $\mathbf{C}$, respectively). All liver samples were obtained from mice treated with 600 $\mathrm{mg} / \mathrm{kg} \mathrm{APAP}$, followed by treatment with L803-mts ( $800 \mathrm{nmol}$ per mouse) or phosphate-buffered saline (PBS). L803-mts or PBS was administered 4 hours after APAP, followed by every 24 hours until sample collection. All samples were collected at 18, 24, 48, and 72 hours after APAP treatment. ${ }^{*} P<0.05$ versus APAP + PBS. A, APAP; GAPDH, glyceraldehyde-3phosphate dehydrogenase; GSK3i, GSK3 inhibitor; I, inhibitor.

Previous studies from our laboratory have demonstrated the role of $\beta$-catenin signaling in liver regeneration after APAP overdose. ${ }^{6}$ Consistent with our previous report, ${ }^{6}$ $\beta$-catenin was phosphorylated at GSK3 target sites (inactivation sites) after a higher dose of APAP, where liver regeneration was attenuated and delayed. This suggested a plausible role of GSK3 activity in impaired liver regeneration after severe APAP-induced hepatotoxicity and inhibition of GSK3 could result in improved liver regeneration. Consistent with this hypothesis, pharmacological inhibition of GSK3 using highly selective inhibitor of GSK3 caused dose-dependent early hepatocyte proliferation and liver regeneration after APAP-induced hepatotoxicity. These data indicate the inhibitory role of GSK3 on liver regeneration after APAP overdose. The proproliferative role of GSK3 inhibition is consistent with a previous report, where inhibitor of GSK3 induced in vitro expansion of hepatocytes. ${ }^{18}$ However, our finding is contradictory to few previous reports where inhibition or siRNA-mediated downregulation of GSK3 resulted in impaired hepatocyte proliferation after $\mathrm{PH}^{24-26}$ These discrepancies in findings may be because of inherent differences in models used to study liver regeneration. APAP overdose has a significant mechanistic difference from $\mathrm{PH}$, including presence of significant cell death, liver injury, oxidative stress, and asynchronous nature of cell cycle. Our study highlights the notion that the same signaling pathway may have a different and sometimes opposite role in different models of regeneration. These studies also underline the importance of investigating mechanisms of liver regeneration after APAP overdose separately from $\mathrm{PH}$ to determine the inherent modelassociated differences.

We further investigated the potential mechanisms underlying the role of GSK3 inactivation in causing early initiation of liver regeneration after APAP-induced hepatotoxicity. Inhibition of GSK3 caused an early increase in cyclin D1 expression. Thus, early initiation of liver regeneration after GSK3 inhibition was most possibly because of early induction of cyclin D1 (which is a critical regulator of cell cycle entry) and subsequent phosphorylation of $\mathrm{Rb}$ protein. Interestingly, cyclin $\mathrm{D} 1$ expression was consistently increased after GSK3 inhibition at all of the investigated time points. However, PCNA expression and cell proliferation were higher only at the earlier time points after GSK3 inhibition and were comparable to the control group at the later time points. A moderate increase in cyclin D1 expression at later time points in the control group might be responsible for a delayed increase in PCNA expression and cell proliferation in the control group. Furthermore, potential activation of cell cycle inhibitory pathways at later time points after GSK3 inhibition might be involved in a plateauing effect on cell proliferation, which needs further comprehensive investigation. We investigated protein levels of p21 (cell cycle inhibitor), which were found to be comparable between two groups (data not shown). Another possibility is cellular stress associated with ongoing liver injury, which was not affected by GSK3 inhibition. It is possible that this cellular stress may further inhibit cell cycle progression and PCNA expression. Nevertheless, an early increase in cyclin D1 expression was correlated with early initiation of liver regeneration after GSK3 inhibition. Furthermore, our results demonstrate that early activation of $\beta$-catenin after GSK3 inhibition might be involved in early induction in cyclin D1 and accelerate liver regeneration after APAP-induced liver injury. This corroborated our previous findings that overexpression of the stable form of $\beta$-catenin signaling in mice increased cyclin D1 expression and improved liver regeneration ${ }^{6}$; and $\beta$-catenin knockout mice 


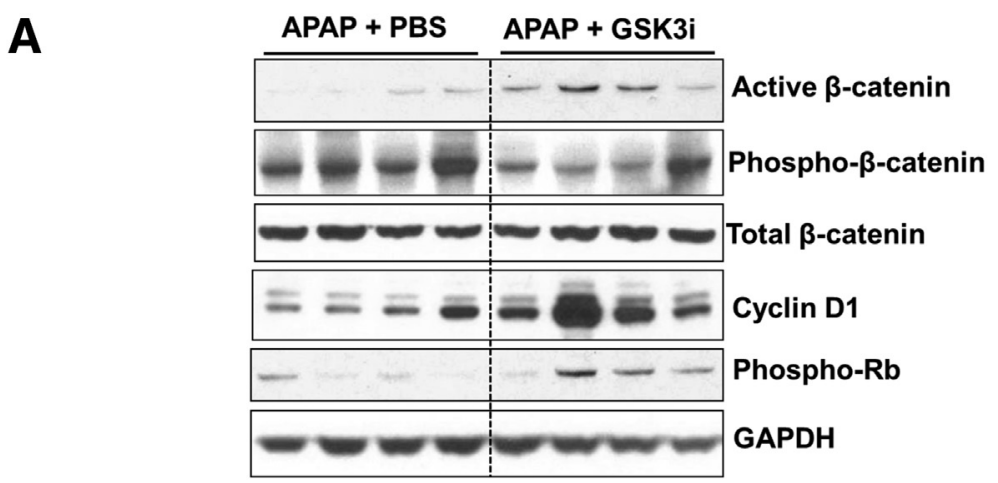

B

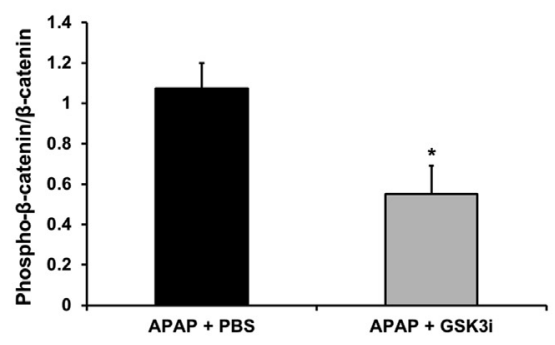

C

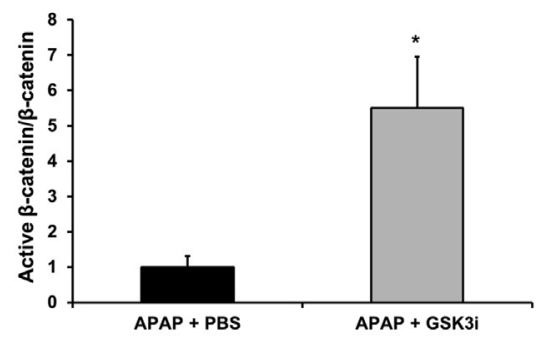

E

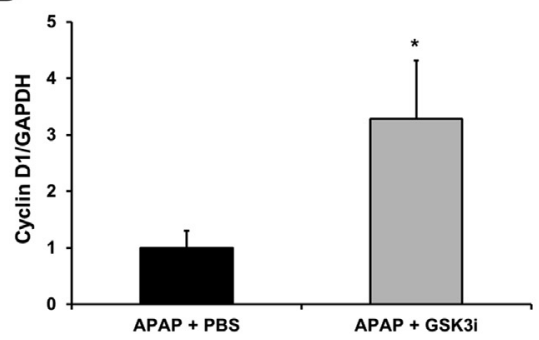

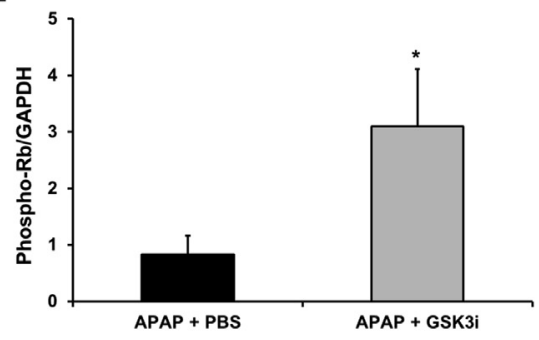

Figure 6 Inhibition of GSK3 resulted in increased activation of $\beta$-catenin after APAP overdose. Western blot analysis of phospho- $\beta$ catenin (Thr41/Ser33/Ser37), active $\beta$-catenin, total $\beta$-catenin, cyclin D1, and phospho-Rb in total cell lysate $(\mathbf{A})$, with their densitometric analysis shown (B-E, respectively). All liver samples were obtained from mice treated with $600 \mathrm{mg} / \mathrm{kg} \mathrm{APAP}$, followed by treatment with L803-mts (800 nmol per mouse) or phosphate-buffered saline (PBS). L803-mts or PBS was administered 4 hours after APAP, and samples were collected at 18 hours after APAP treatment. ${ }^{*} P<0.05$ versus APAP + PBS. $\mathrm{GAPDH}$, glyceraldehyde-3-phosphate dehydrogenase; GSK3i, GSK3 inhibitor. showed decreased liver regeneration after APAP-induced liver injury. ${ }^{9}$ Similar to our findings in the APAP model, overexpression of $\beta$-catenin in mice resulted in early initiation of liver regeneration in the $\mathrm{PH}$ model, with increased expression of cyclin D1 without altering peak liver regeneration. $^{21}$ Similarly, liver-specific $\beta$-catenin deletion in mice caused delayed liver regeneration after $\mathrm{PH}$, which was correlated with decreased cyclin D1 induction. ${ }^{22,23}$ Apart from negative regulation of $\beta$-catenin, GSK3 can directly regulate several other cell proliferation mediators, including cyclin D1, c-Myc, c-Jun, and eIF2B. ${ }^{14,17}$ Thus, GSK3 could also potentially alter liver regeneration after APAP overdose in a $\beta$-catenin-independent manner, which remained to be investigated.

Shinohara et $\mathrm{al}^{27}$ recently reported that an attenuation of APAP hepatotoxicity on antisense oligonucleotides mediated silencing of GSK3 $\beta$. This was attributed to the role of GSK $3 \beta$ in liver injury initiation through c-Jun N-terminal kinase activation and myeloid cell leukemia-1 protein degradation. In our study, treatment with GSK3i did not alter APAP-induced hepatotoxicity at any of the investigated time points. Furthermore, we did not observe any alteration of APAP-mediated c-Jun amino terminal kinase activation by GSKi treatment (data not shown). These discrepancies are because of differences in experimental design. In our study, GSK3i was administered 4 hours after APAP, such that injury initiation events have already taken place, whereas a pretreatment strategy was used in the previous study. ${ }^{27}$ In fact, we purposefully used a posttreatment strategy such that APAP injury initiation was not interfered. This is because any alteration in injury can have secondary effects of liver regeneration and the purpose of the study was to study the direct role of GSK3 in liver regeneration. Thus, our study indicated that the effect of accelerated liver regeneration is directly mediated by GSK3 inhibition and is not secondary to altered injury.

In the past, the primary focus of research in the area of APAP-inflicted liver pathophysiology has been mechanisms of initiation of APAP hepatotoxicity and targeting liver injury for development of therapeutic strategies. However, many of the APAP overdose patients typically seek medical attention late after APAP overdose, such that injury is 
already established. ${ }^{5}$ Even after decades of research, $\mathrm{N}$-acetylcysteine is still the only standard therapy for APAP overdose that is effective only at an early stage. ${ }^{3}$ In mice, $\mathrm{N}$-acetylcysteine does not provide protection when administered 4 hours after APAP. ${ }^{33}$ Liver regeneration is a parameter that can be potentially modulated even at the late stage in the APAP-induced ALF and, thus, is an attractive therapeutic target. In our study, initiating GSK3i treatment 4 hours after APAP overdose in mice showed improved liver regeneration parameters and some indication of delay in mortality. The time window of therapeutic effectiveness of GSK3i is expected to be much more in humans considering that the pathogenesis of APAP hepatotoxicity is generally delayed in humans compared to mice. ${ }^{34}$ Our study suggested the potential benefit of GSK3 inhibition in improving liver regeneration even after a delayed intervention after APAP overdose. Thus, future investigations on similar lines are warranted to identify novel therapeutic targets to stimulate live regeneration after APAP-induced hepatotoxicity, which are currently underexplored. Regenerative therapies at least could be helpful in bridging the time gap before liver is available for transplantation to ALF patients.

In conclusion, our study revealed the novel inhibitory role of GSK3 in timely liver regeneration response after APAPinduced hepatotoxicity. Inhibition of GSK3-accelerated liver regeneration after APAP overdose associated with early induction of cyclin D1 and increased $\beta$-catenin signaling. Thus, our study suggested inhibition of GSK3 as a potential therapeutic strategy to improve liver regeneration after APAP-induced ALF.

\section{Supplemental Data}

Supplemental material for this article can be found at http://dx.doi.org/10.1016/j.ajpath.2016.11.014.

\section{References}

1. Lee WM, Squires RH Jr, Nyberg SL, Doo E, Hoofnagle JH: Acute liver failure: summary of a workshop. Hepatology 2008, 47: $1401-1415$

2. Lee WM: Acute liver failure. Semin Respir Crit Care Med 2012, 33: $36-45$

3. Bernal W, Lee WM, Wendon J, Larsen FS, Williams R: Acute liver failure: a curable disease by 2024? J Hepatol 2015, 62:S112-S120

4. Athuraliya TN, Jones AL: Prolonged N-acetylcysteine therapy in late acetaminophen poisoning associated with acute liver failure: a need to be more cautious? Crit Care 2009, 13:144

5. Larson AM: Acetaminophen hepatotoxicity. Clin Liver Dis 2007, 11: 525-548. vi

6. Bhushan B, Walesky C, Manley M, Gallagher T, Borude P, Edwards G, Monga SP, Apte U: Pro-regenerative signaling after acetaminophen-induced acute liver injury in mice identified using a novel incremental dose model. Am J Pathol 2014, 184: 3013-3025

7. Bhushan B, Edwards G, Desai A, Michalopoulos GK, Apte U: Liverspecific deletion of integrin-linked kinase in mice attenuates hepatotoxicity and improves liver regeneration after acetaminophen overdose. Gene Expr 2016, 17:35-45

8. Bhushan B, Borude P, Edwards G, Walesky C, Cleveland J, Li F, Ma X, Apte U: Role of bile acids in liver injury and regeneration following acetaminophen overdose. Am J Pathol 2013, 183: $1518-1526$

9. Apte U, Singh S, Zeng G, Cieply B, Virji MA, Wu T, Monga SP: Betacatenin activation promotes liver regeneration after acetaminopheninduced injury. Am J Pathol 2009, 175:1056-1065

10. Schmidt LE, Dalhoff K: Alpha-fetoprotein is a predictor of outcome in acetaminophen-induced liver injury. Hepatology 2005, 41:26-31

11. Hu B, Colletti LM: Stem cell factor and c-kit are involved in hepatic recovery after acetaminophen-induced liver injury in mice. Am J Physiol Gastrointest Liver Physiol 2008, 295:G45-G53

12. Donahower BC, McCullough SS, Hennings L, Simpson PM, Stowe CD, Saad AG, Kurten RC, Hinson JA, James LP: Human recombinant vascular endothelial growth factor reduces necrosis and enhances hepatocyte regeneration in a mouse model of acetaminophen toxicity. J Pharmacol Exp Ther 2010, 334:33-43

13. Michalopoulos GK: Liver regeneration. J Cell Physiol 2007, 213 $286-300$

14. Kaidanovich-Beilin O, Woodgett JR: GSK-3: functional insights from cell biology and animal models. Front Mol Neurosci 2011, 4:16

15. Jope RS, Johnson GV: The glamour and gloom of glycogen synthase kinase-3. Trends Biochem Sci 2004, 29:95-102

16. Doble BW, Woodgett JR: GSK-3: tricks of the trade for a multi-tasking kinase. J Cell Sci 2003, 116:1175-1186

17. Sutherland C: What are the bona fide GSK3 substrates? Int J Alzheimers Dis 2011, 2011:505607

18. Ito H, Kamiya A, Ito K, Yanagida A, Okada K, Nakauchi H: In vitro expansion and functional recovery of mature hepatocytes from mouse adult liver. Liver Int 2012, 32:592-601

19. Gotschel F, Kern C, Lang S, Sparna T, Markmann C, Schwager J, McNelly S, von Weizsacker F, Laufer S, Hecht A, Merfort I: Inhibition of GSK3 differentially modulates NF-kappaB, CREB, AP-1 and betacatenin signaling in hepatocytes, but fails to promote TNF-alphainduced apoptosis. Exp Cell Res 2008, 314:1351-1366

20. Nejak-Bowen KN, Monga SP: Beta-catenin signaling, liver regeneration and hepatocellular cancer: sorting the good from the bad. Semin Cancer Biol 2011, 21:44-58

21. Nejak-Bowen KN, Thompson MD, Singh S, Bowen WC Jr, Dar MJ, Khillan J, Dai C, Monga SP: Accelerated liver regeneration and hepatocarcinogenesis in mice overexpressing serine-45 mutant betacatenin. Hepatology 2010, 51:1603-1613

22. Tan X, Behari J, Cieply B, Michalopoulos GK, Monga SP: Conditional deletion of beta-catenin reveals its role in liver growth and regeneration. Gastroenterology 2006, 131:1561-1572

23. Sekine S, Gutierrez PJ, Lan BY, Feng S, Hebrok M: Liver-specific loss of beta-catenin results in delayed hepatocyte proliferation after partial hepatectomy. Hepatology 2007, 45:361-368

24. Sekiya S, Suzuki A: Glycogen synthase kinase 3 beta-dependent Snail degradation directs hepatocyte proliferation in normal liver regeneration. Proc Natl Acad Sci U S A 2011, 108:11175-11180

25. Jin J, Wang GL, Shi X, Darlington GJ, Timchenko NA: The ageassociated decline of glycogen synthase kinase 3beta plays a critical role in the inhibition of liver regeneration. Mol Cell Biol 2009, 29: $3867-3880$

26. Chen H, Yang S, Yang Z, Ma L, Jiang D, Mao J, Jiao B, Cai Z: Inhibition of GSK-3beta decreases NF-kappaB-dependent gene expression and impairs the rat liver regeneration. J Cell Biochem 2007, 102: $1281-1289$

27. Shinohara M, Ybanez MD, Win S, Than TA, Jain S, Gaarde WA, Han D, Kaplowitz N: Silencing glycogen synthase kinase-3beta inhibits acetaminophen hepatotoxicity and attenuates JNK activation and loss of glutamate cysteine ligase and myeloid cell leukemia sequence 1. J Biol Chem 2010, 285:8244-8255 
28. Plotkin B, Kaidanovich O, Talior I, Eldar-Finkelman H: Insulin mimetic action of synthetic phosphorylated peptide inhibitors of glycogen synthase kinase-3. J Pharmacol Exp Ther 2003, 305:974-980

29. Wolfe A, Thomas A, Edwards G, Jaseja R, Guo GL, Apte U: Increased activation of the Wnt/beta-catenin pathway in spontaneous hepatocellular carcinoma observed in farnesoid $\mathrm{X}$ receptor knockout mice. $\mathrm{J}$ Pharmacol Exp Ther 2011, 338:12-21

30. Sun L, Beggs K, Borude P, Edwards G, Bhushan B, Walesky C, Roy N, Manley MW Jr, Gunewardena S, O’Neil M, Li H, Apte U: Bile acids promote diethylnitrosamine-induced hepatocellular carcinoma via increased inflammatory signaling. Am J Physiol Gastrointest Liver Physiol 2016, 311:G91-G104
31. Rao R, Hao CM, Redha R, Wasserman DH, McGuinness OP, Breyer MD: Glycogen synthase kinase 3 inhibition improves insulinstimulated glucose metabolism but not hypertension in high-fat-fed C57BL/6J mice. Diabetologia 2007, 50:452-460

32. Fausto N: Liver regeneration. J Hepatol 2000, 32:19-31

33. James LP, McCullough SS, Lamps LW, Hinson JA: Effect of $\mathrm{N}$-acetylcysteine on acetaminophen toxicity in mice: relationship to reactive nitrogen and cytokine formation. Toxicol Sci 2003, 75:458-467

34. Xie Y, McGill MR, Dorko K, Kumer SC, Schmitt TM, Forster J, Jaeschke H: Mechanisms of acetaminophen-induced cell death in primary human hepatocytes. Toxicol Appl Pharmacol 2014, 279: $266-274$ 